\title{
Meyer's Concept of Quasicrystal and Quasiregular Sets
}

\author{
J.C. Lagarias \\ AT\&T Bell Laboratories, Murray Hill, New Jersey 07974 USA. E-mail: jcl@research att com
}

Received: 19 June 1995/Accepted: 14 November 1995

\begin{abstract}
This paper relates two mathematical concepts of long-range order of a set of atoms $\Lambda$, each of which is based on restrictions on the set of interatomic distances $\Lambda-\Lambda$. A set $\Lambda$ in $\mathbb{R}^{n}$ is a Meyer set if $\Lambda$ is a Delone set and there is a finite set $F$ such that $\Lambda-\Lambda \subseteq \Lambda+F$. Y. Meyer proposed that such sets include the possible structures of quasicrystals. He obtained a structure theory for such sets, which reformulates results that he obtained in harmonic analysis around 1970, and which relates these sets to cut-and-project sets. In geometric crystallography V.I. Galiulin introduced the concept of quasiregular set, which is a set $\Lambda$ such that both $\Lambda$ and $\Lambda-\Lambda$ are Delone sets. This paper shows that these two concepts are identical.
\end{abstract}

\section{Introduction}

In 1984 Schechtman et al. [40] discovered materials whose X-ray diffraction spectra had sharp spots indicative of long range order, which exhibited non-crystallographic symmetries. Such materials cannot have a periodic arrangement of atoms; they are now called quasicrystals, cf. [27]. In the last ten years there has been an immense amount of theoretical and experimental research effort to determine the atomic structure of such materials and to find mechanisms that explain how they form and remain energetically stable, cf. [19].

On the theoretical side, a large class of structures have been constructed which exhibit sharp spots in their X-ray diffraction spectra and which have noncrystallographic symmetries. These sets consist of cut-and-project sets of points ([1], [9], [10], [23], [26], [27]). Alternate descriptions of possible quasicrystalline structures were also given in terms of quasiperiodic tilings using a finite set of prototiles ([13], [22], [29]). Many of these tiling constructions trace back to work of de Bruijn on Penrose tilings [2]. Another direction of work concerns "local rules" as analogues of short-range interactions that might explain the formation of such structures ([20], [24], [25], [28], [34], [41]). It appears that there are some quasicrystalline tilings not describable by "local rules" of particular types [4]. 
This paper studies two mathematical characterizations of structures with long range order which are sufficiently broad to include the possible structures of quasicrystalline materials. Mathematical idealizations of quasicrystals model them as discrete sets of points ("atoms") and consider infinitely large structures in arbitrary dimensions. The basic assumption of "condensed matter" is embodied in the following definition.

Definition 1.1. A set $\Lambda$ in $\mathbb{R}^{n}$ is a Delone set, (or Delaunay set or (r, $\mathbf{R}$ )-set) if it has the two properties:

(i) Uniformly Discrete. There is a distance $r>0$ such that the ball of radius $r$ centered about any $\mathbf{x} \in \mathbb{R}^{n}$ contains at most one point of $\Lambda$.

(ii) Relatively Dense. There is a distance $R>0$ such that the ball of radius $R$ centered about any $\mathbf{x} \in \mathbb{R}^{n}$ contains at least one point of $\Lambda$.

This concept is named after B.N. Delone [6,7] (alternative spelling: B.N. Delaunay), see also $[14,15]$.

The concept of a crystal is that of a discrete point set having a full lattice of translation symmetries.

Definition 1.2. An ideal crystal $\Lambda$ in $\mathbb{R}^{n}$ is a point set of the form

$$
L+F:=\{\mathbf{x}+\mathbf{f}: \mathbf{x} \in L \text { and } \mathbf{f} \in F\}
$$

in which $L$ is a (full rank) lattice in $\mathbb{R}^{n}$ and $F$ is a finite set.

There has been a search for suitable generalizations of "ideal crystal" that still prescribe long-range order but include the possible atomic structures of quasicrystalline materials. Among these, two generalizations have been proposed which are based on restrictions on the set of "interatomic distances"

$$
\Lambda-\Lambda:=\left\{\mathbf{x}-\mathbf{x}^{\prime}: \mathbf{x}, \mathbf{x}^{\prime} \in \Lambda\right\} .
$$

The set $\Lambda-\Lambda$ is relevant to atomic structure because the far-field X-ray diffraction pattern of $\Lambda$ is the Fourier transform of the points of $\Lambda-\Lambda$ taken in a suitable limiting sense, i.e. it is the Fourier transform of the two-point correlation function (autocorrelation function) of $\Lambda$, cf. Hof $[16,17]$ and Senechal [39].

The first generalization of ideal crystal is a weakening of the notion of regular point system, on which geometric crystallography is based, see Sect. 2. This generalization was proposed by Galiulin [15] in 1989.

Definition 1.3. $A$ quasiregular set is a Delone set $\Lambda$ such that $\Lambda-\Lambda$ is also a Delone set.

This definition can also be viewed as a relaxation of the concept of a set prescribed by "local rules," as explained in Sect. 2 .

A second generalization of ideal crystal is due to Y. Meyer [30], who proposed it in connection with questions in harmonic analysis ([30,31]). Meyer [32] terms these sets "quasicrystals," while Moody [33] calls them "Meyer sets."

Definition 1.4. $A$ Meyer set $\Lambda$ is a Delone set such that $\Lambda-\Lambda \subseteq \Lambda+F$ for some finite set $F$.

Meyer gave several equivalent definitions of this class of sets, which we summarize in Sect. 2. Meyer sets are a generalization of "cut-and-project" sets, and have a 
harmonic-analytic characterization which is analogous to an X-ray diffraction pattern having a series of bright spots.

The main result of this paper is:

Theorem 1.1. The following properties of a set $\Lambda$ in $\mathbb{R}^{n}$ are equivalent.

(i) $\Lambda$ is a Meyer set.

(ii) $\Lambda$ is a quasiregular set.

Meyer [32] observed that $\Lambda$ is a Meyer set if and only if $\Lambda$ and $\Lambda-\Lambda-\Lambda$ are both Delone sets, and this shows that (i) $\Rightarrow$ (ii). The new result is (ii) $\Rightarrow$ (i). For completeness we include an easy proof that (i) $\Rightarrow$ (ii).

For general background we give in Sect. 2 a discussion of quasiregular sets and Meyer sets and their relation to other concepts of "crystal" and "quasicrystal". The proof of Theorem 1.1 follows in Sect. 3, and does not depend on any result of Sect. 2.

In Sect. 4 we use Theorem 1.1 to derive an improved harmonic-analytic criterion for a set $\Lambda$ to be a Meyer set (Theorem 4.1). This result is due to Robert Moody.

The notion of Meyer set provides a mathematical framework that seems to be sufficient to include most reasonable quasicrystalline structures. In Sect. 5 we indicate how tiling models of quasicrystals fit into this framework. We also observe in Sect. 5 that there exist Meyer sets that are not quasicrystalline in any reasonable sense, because they have no well-defined autocorrelation function (X-ray diffraction pattern). Extra conditions might be imposed on Meyer sets to obtain a narrower concept of "quasicrystal" and we indicate some possibilities.

\section{Quasiregular Sets and Meyer Sets}

The notion of quasiregular set derives from geometric crystallography, which according to Engel $[11,12]$ is the study of regular point systems.

Definition 2.1. A set $\Lambda$ in $\mathbb{R}^{n}$ is a regular point system if it is a Delone set and if it "looks the same" from every point $\mathbf{x} \in \Lambda$ in the sense that for any $\mathbf{x}_{1}, \mathbf{x}_{2} \in \Lambda$ the sets $\Lambda-\mathbf{x}_{1}$ and $\Lambda-\mathbf{x}_{2}$ are congruent to each other by an isometry of $\mathbb{R}^{n}$ that fixes $\mathbf{0}$. (An isometry that fixes $\mathbf{0}$ is either a rotation or a reflection followed by a rotation.)

This definition was given in 1874 by Sohncke [42], who classified the regular point systems in two dimensions. It is known that in each dimension $n \geqq 1$, every regular point system in $\mathbb{R}^{n}$ is an ideal crystal. In 1976 Delone et al. [7] showed that regularity can be specified by "local rules."

Definition 2.2. A set of local rules, $\mathscr{L}$, is a set of finite point configurations, each of which is contained in the ball $B\left(\mathbf{0}, R^{\prime}\right)$ of radius $R^{\prime}$ around $\mathbf{0}$. A Delaunay set $\Lambda$ satisfies the local rules $\mathscr{L}$ under translations if for every $\mathbf{x} \in \Lambda$,

$$
(\Lambda-\mathbf{x}) \cap B\left(\mathbf{0}, R^{\prime}\right) \in \mathscr{L}
$$

More generally $\Lambda$ satisfies the local rules $\mathscr{L}$ under isometries if for every $\mathbf{x}$ in $\Lambda$, the set $(\Lambda-\mathbf{x}) \cap B\left(\mathbf{0}, R^{\prime}\right)$ is congruent to some element of $\mathscr{L}$ under an isometry that fixes $\mathbf{0}$. We call $R^{\prime}$ the range of the set of local rules. 
To state the result of Delone et al. [7], let $B(\mathbf{x}, R)=\{\mathbf{y}:\|\mathbf{y}-\mathbf{x}\| \leqq R\}$ denote the closed ball of Euclidean radius $R$ around $\mathbf{x}$.

Theorem 2.1. Let $\Lambda$ be an $(r, R)$-set in $\mathbb{R}^{n}$. There is a constant $c$ depending on the ratio $R / r$ and on $n$ such that if all the neighborhoods $(\Lambda-\mathbf{x}) \cap B(\mathbf{0}, c R)$ for $\mathbf{x} \in \Lambda$ are congruent under isometries of $\mathbb{R}^{n}$ that fix $\mathbf{0}$, then $\Lambda$ is a regular point system.

In this case the set $\mathscr{L}$ of local rules under isometries consists of a single configuration. Engel [11] gives examples of $(r, R)$ sets $\Lambda$ which are not regular point systems but which have the property that all sets $(\Lambda-\mathbf{x}) \cap B(\mathbf{0}, 4 R)$ for $\mathbf{x} \in \Lambda$ are isometric. Engel [11, Theorem 9.9] claims a stronger form of Theorem 1.1 that takes $c=6$ independent of both $R / r$ and $n$, but his proof is incomplete, and depends on his Conjecture 9.7.

Not every ideal crystal is a regular point system. However it is fairly easy to extend Theorem 2.1 to show that any ideal crystal $\Lambda=L+F$ is determined by a finite set of "local rules" under isometries or under translations, cf. [8].

To allow nonperiodic structures Galiulin [14] suggested relaxing the concept of a regular point system to that of a quasiregular set given in Sect. 1, i.e. to require only that $\Lambda$ and $\Lambda-\Lambda$ both be Delone sets. Quasiregular sets retain a weak vestige of "local rules" in that they possess the locally finite atlas property: for each $R>0$ there are only finitely many distinct sets among all sets $(\Lambda-\mathbf{x}) \cap B(\mathbf{0}, R)$, with $\mathbf{x} \in \Lambda$. (The collection of all sets $(\Lambda-\mathbf{x}) \cap B(\mathbf{0}, R)$ for $\mathbf{x} \in \Lambda$ is called the $R$-atlas of 1 .)

The concept of Meyer set was originally defined in terms of a notion of duality in harmonic analysis which is related to almost-periodicity.

Definition 2.3. Given a Delone set $\Lambda \subseteq \mathbb{R}^{n}$, for any $\varepsilon>0$ its $\varepsilon$-dual $\Lambda^{\varepsilon}$ is given by

$$
\Lambda^{\varepsilon}:=\left\{\boldsymbol{\mu} \in \mathbb{R}^{n}:|\exp (2 \pi i\langle\boldsymbol{\mu}, \mathbf{x}\rangle)-1| \leqq \varepsilon \text { for all } \mathbf{x} \in \Lambda\right\}
$$

where $\langle\boldsymbol{\mu}, \mathbf{x}\rangle:=\sum_{i=1}^{n} \mu_{i} x_{i}$.

This concept generalizes duality of lattices, for in the case that $L$ is a full rank lattice in $\mathbb{R}^{n}$, the set $L^{\varepsilon}$ is equal to the dual lattice $L^{*}:=\left\{\boldsymbol{\mu} \in \mathbb{R}^{n}:\langle\boldsymbol{\mu}, \mathbf{x}\rangle \in \mathbb{Z}\right.$ for all $\mathbf{x} \in L\}$ for $0 \leqq \varepsilon<\sqrt{3}$. However for most Delone sets $\Lambda$ the $\varepsilon$-dual $\Lambda^{\varepsilon}=\{\mathbf{0}\}$ for $0<\varepsilon<2$. We are concerned with those $\Lambda$ such that $\Lambda^{\varepsilon}$ is "large."

Definition 2.4. $A$ discrete set $\Lambda$ is $\varepsilon$-harmonious if its $\varepsilon$-dual $\Lambda^{\varepsilon}$ is relatively dense. It is harmonious if $\Lambda$ is $\varepsilon$-harmonious for all $\varepsilon>0$.

Meyer's original concept was of a relatively dense harmonious set, cf. Theorem 2.2 below. These sets are closely related to cut-and-project sets, which Meyer introduced in 1972 under the name "model set," see [31], p. 48.

Definition 2.5. The cut-and-project set $\Lambda(L, \Omega)$ in $\mathbb{R}^{n}$ is associated to data $(L, \Omega)$, in which $L$ is a full rank lattice in $\mathbb{R}^{r}=\mathbb{R}^{n} \times \mathbb{R}^{k}$ with $k \geqq 0$, which satisfies

(i) No two elements of $L$ have the same image under orthogonal projection $\pi_{\|}$onto $\mathbb{R}^{n}$,

(ii) The image of $L$ under orthogonal projection $\pi_{\perp}$ onto $\mathbb{R}^{k}$ is dense in $\mathbb{R}^{k}$, and $\Omega$ is a "window" in $\mathbb{R}^{k}$ which is a nonempty bounded open set. Then $\Lambda(L, \Omega)$ 
is given by

$$
\Lambda(L, \Omega):=\left\{p_{\|}(\mathbf{x}): \mathbf{x} \in L \text { and } p_{\perp}(\mathbf{x}) \in \Omega\right\} .
$$

Meyer showed that the Fourier transform of a sum of delta functions at points of a cut-and-project set $\Lambda(L, \Omega)$ is a weighted sum of delta functions on a countable set, and if in addition the window $\Omega$ is convex and symmetric around $\mathbf{0}$ then the largest weights are at points of its $\varepsilon$-dual $\Lambda^{\varepsilon}$ for small $\varepsilon$, cf. [32], Theorem 7 . The $\mathrm{X}$-ray diffraction spectrum of cut-and-project sets is also supported on a countable set, cf. Elser [10].

Meyer [31] proved the following relations among these concepts.

Theorem 2.2 (Meyer). The following properties of a set $\Lambda$ in $\mathbb{R}^{n}$ are equivalent.

(1) $\Lambda$ is a Meyer set.

(2) $\Lambda$ is a Delone set and there exists a cut-and-project set $\Lambda(L, \Omega)$ and a finite set $F$ such that

$$
\Lambda \subseteq \Lambda(L, \Omega)+F
$$

(3) $\Lambda$ is a relatively dense harmonious set. That is, $\Lambda$ is a relatively dense set such that for each $\varepsilon>0$ its $\varepsilon$-dual $\Lambda^{\varepsilon}$ is relatively dense.

Note that criterion (2) implies that all cut-and-project sets are Meyer sets. However there apparently are Meyer sets that do not contain any cut-and-project set. The criterion (3) is related to X-ray diffraction in that for directions $\boldsymbol{\mu} \in \Lambda^{\varepsilon}$ the phases of the points of $\Lambda$ approximately line up so that the X-ray diffraction pattern of $\Lambda$ (if it exists) will have high intensity at such points. We show in Sect. 4 that criterion (3) can be weakened.

Meyer's proof of Theorem 2.2 is quite intricate: see [31], Theorem I, p. 43 and Theorem IV, p. 48 for (2) $\Leftrightarrow(3)$, and Theorem X, p. 71 for (1) $\Leftrightarrow(3)$. More results on Meyer sets appear in [32,34].

Recently Moody, Patera and others have found local growth mechanisms to describe the formation of certain Meyer sets, see [5, 24, 34].

\section{Equivalence of Meyer Sets and Quasiregular Sets}

We prove Theorem 1.1. Let $\|\mathbf{x}\|=\left(\sum_{i=1}^{n} x_{i}^{2}\right)^{1 / 2}$ denote the Euclidean norm on $\mathbb{R}^{n}$.

Proof of $(i) \Rightarrow($ ii $)$. We suppose that $\Lambda$ is a Delone set with $\Lambda-\Lambda \subseteq \Lambda+F$, and we are to show that $\Lambda-\Lambda$ is a Delone set. Now $\Lambda-\Lambda$ contains a translate of $\Lambda$, say $\Lambda-\{\lambda\}$, so it inherits the relative denseness property from $\Lambda$.

It remains to show that $\Lambda-\Lambda$ is uniformly discrete, which we do by showing that $\Lambda+F$ is uniformly discrete. (This is not true for general Delone sets $\Lambda$, e.g. take $\Lambda=\left\{n+\frac{1}{|n|+2}: n \in \mathbb{Z}\right\}$ and $F=\{0,1\}$.) We argue by contradiction. A set $S$ is discrete if $\#\{\mathbf{s} \in S:\|\mathbf{s}\|<T\}$ is finite for all $T>0$. Certainly $\Lambda+F$ is discrete because $\Lambda$ is discrete and $F$ is finite. If $\Lambda+F$ were not uniformly discrete, then we can find a sequence of vectors of differences of elements of $\Lambda+F$, say

$$
\mathbf{x}_{k}:=\left(\lambda_{i_{k}}+\mathbf{f}_{k}\right)-\left(\lambda_{j_{k}}+\mathbf{f}_{k}^{\prime}\right) \rightarrow \mathbf{0},
$$

which are all distinct and become arbitrarily small as $k \rightarrow \infty$. Extract a subsequence $\mathbf{x}_{l}$ on which the pair $\left(\mathbf{f}_{k}, \mathbf{f}_{k}^{\prime}\right)$ is constant. Then

$$
\mathbf{y}_{l}:=\lambda_{i_{l}}-\lambda_{j_{l}} \rightarrow \mathbf{f}_{k}^{\prime}-\mathbf{f}_{k},
$$


and the $\mathbf{y}_{l}$ assume infinitely many distinct values. Thus the sequence $\left\{\mathbf{y}_{l}\right\}$ has a finite limit point, which shows that $\Lambda-\Lambda$ is not discrete. But $\Lambda+F$ contains $\Lambda-\Lambda$, hence $\Lambda+F$ is not discrete, which is a contradiction.

Proof of $(i i) \Rightarrow(i)$. We suppose that $\Lambda$ is an $(r, R)$-set and that $\Lambda-\Lambda$ is an $\left(r^{\prime}, R^{\prime}\right)$ set, and we are to find a finite set $F$ such that $\Lambda-\Lambda \subseteq \Lambda+F$. Without loss of generality we may suppose that $\mathbf{0} \in \Lambda$, by translating $\Lambda$ to $\Lambda-\mathbf{x}$, which leaves $\Lambda-\Lambda$ unchanged, and simultaneously replacing $F$ by $F+\mathbf{x}$.

We first assume only that $\Lambda-\Lambda$ is discrete, and show that this implies that the additive group $\mathbb{Z}[\Lambda-\Lambda]$ generated by the elements of $\Lambda-\Lambda$ is finitely generated. The discreteness of $\Lambda-\Lambda$ implies that the set

$$
\mathscr{S}:=\{\mathbf{x} \in \Lambda-\Lambda:\|\mathbf{x}\|<3 R\},
$$

is finite. Indeed the open balls of radius $\frac{r^{\prime}}{2}$ around each $\mathbf{w} \in \mathscr{S}$ are all disjoint and lie inside the ball of radius $3 R+\frac{r^{\prime}}{2}$, which gives obtain the volume bound

$$
|\mathscr{S}| \leqq\left(\frac{6 R}{r^{\prime}}+1\right)^{n}
$$

The vectors in $\mathscr{S}$ generate a finite-dimensional vector space over the rationals $\mathbb{Q}$, denoted

$$
V:=\mathbb{Q}[\mathbf{x}: \mathbf{x} \in \mathscr{S}]
$$

Let $\left[\mathbf{y}_{1}^{\prime}, \ldots, \mathbf{y}_{s}^{\prime}\right]$ be a subset of $\mathscr{S}$ forming a basis of this vector space. ${ }^{1}$ Every vector $\mathbf{x}_{i} \in \mathscr{S}$ can be expressed as

$$
\mathbf{x}_{i}:=\sum_{j=1}^{s} r_{i j} \mathbf{y}_{j}^{\prime}, \quad \text { with } r_{i j} \in \mathbb{Q} .
$$

Let $D$ be the lowest common denominator of all the $r_{i j}$, i.e. $r_{i j}=\frac{k_{i j}}{D}$ with $k_{i j} \in \mathbb{Z}$ and choose the new basis $\left[\mathbf{y}_{1}, \ldots, \mathbf{y}_{s}\right]$ given by

$$
\mathbf{y}_{i}:=\frac{1}{D} \mathbf{y}_{i}^{\prime} .
$$

If $\mathbf{y}=\sum_{i=1}^{s} n_{i} \mathbf{y}_{i} \in \mathbb{Z}\left[\mathbf{y}_{1}, \ldots, \mathbf{y}_{s}\right] \subseteq \mathbb{R}^{n}$, then we call $\mathbf{n}=\left(n_{1}, \ldots, n_{s}\right) \in \mathbb{Z}^{s}$ the address of $\mathbf{y}$, and each $\mathbf{y} \in \mathbb{Z}\left[\mathbf{y}_{1}, \ldots, \mathbf{y}_{s}\right]$ has a unique address. Every vector $\mathbf{x}_{i} \in \mathscr{S}$ is an integer linear combination of the $\mathbf{y}_{i}$, namely

$$
\mathbf{x}_{i}=\sum_{k=1}^{s} k_{i j} \mathbf{y}_{j}
$$

hence $\mathscr{S} \subseteq \mathbb{Z}\left[\mathbf{y}_{1}, \ldots, \mathbf{y}_{S}\right]$, and for future reference we set

$$
C_{1}:=\max _{i, j}\left\{\left|k_{i j}\right|\right\}+1 \text {. }
$$

We proceed to show that

$$
\Lambda-\Lambda \subseteq \mathbb{Z}\left[\mathbf{y}_{1}, \ldots, \mathbf{y}_{s}\right]
$$

\footnotetext{
${ }^{1}$ It may be of much higher dimension than $n$.
} 
Let $\mathbf{v} \in \Lambda-\Lambda$, and write $\mathbf{v}=\tilde{\mathbf{w}}-\mathbf{w}$ with $\mathbf{w}, \tilde{\mathbf{w}} \in \Lambda$. Set

$$
\left\{\begin{array}{l}
\mathbf{z}_{k}=\mathbf{w}+k R \frac{\mathbf{v}}{\|\mathbf{v}\|}, \quad \text { for } 0 \leqq k \leqq k_{0}=\left\lfloor\frac{\|\mathbf{v}\|}{R}\right\rfloor, \\
\mathbf{z}_{k_{0}+1}=\tilde{\mathbf{w}}
\end{array}\right.
$$

The $(r, R)$-set property of $\Lambda$ guarantees that there exists $\mathbf{w}_{k} \in \Lambda$ with $\left\|\mathbf{w}_{k}-\mathbf{z}_{k}\right\| \leqq R$ for $0 \leqq k \leqq k_{0}+1$, and we choose $\mathbf{w}_{0}:=\mathbf{w}$ and $\mathbf{w}_{k_{0}+1}=: \tilde{\mathbf{w}}$. The triangle inequality gives

$$
\left\|\mathbf{w}_{k+1}-\mathbf{w}_{k}\right\| \leqq\left\|\mathbf{w}_{k+1}-\mathbf{z}_{k+1}\right\|+\left\|\mathbf{z}_{k+1}-\mathbf{z}_{k}\right\|+\left\|\mathbf{w}_{k}-\mathbf{z}_{k}\right\| \leqq 3 R
$$

for $0 \leqq k \leqq k_{0}$. Thus $\mathbf{w}_{k+1}-\mathbf{w}_{k} \in \mathscr{S} \subseteq \mathbb{Z}\left[\mathbf{y}_{1}, \ldots, \mathbf{y}_{m}\right]$ for $0 \leqq k \leqq k_{0}$, hence

$$
\mathbf{v}=\sum_{k=0}^{k_{0}}\left(\mathbf{w}_{k+1}-\mathbf{w}_{k}\right) \in \mathbb{Z}\left[\mathbf{y}_{1}, \ldots, \mathbf{y}_{s}\right],
$$

which proves (3.3).

Now we use the fact that $\Lambda-\Lambda$ is an $\left(r^{\prime}, R^{\prime}\right)$-set to show that $\Lambda-\Lambda \subseteq \Lambda+F$, where $F$ is the finite set

$$
F:=\left\{\mathbf{z}=l_{1} \mathbf{y}_{1}+\cdots+l_{s} \mathbf{y}_{s}: \text { each } l_{j} \in \mathbb{Z} \text { with }\left|l_{j}\right| \leqq C_{2}\right\},
$$

with $C_{2}=2 C_{1}\left(\frac{4 R}{r^{\prime}}+1\right)^{n}$. To show this, let $\mathbf{v} \in \Lambda-\Lambda$, and write $\mathbf{v}=\tilde{\mathbf{w}}-\mathbf{w}$ for $\mathbf{w}, \tilde{\mathbf{w}} \in \Lambda$. Since $\mathbf{v} \in \mathbb{Z}\left[\mathbf{y}_{1}, \ldots, \mathbf{y}_{s}\right]$, it has a unique address

$$
\mathbf{v}=\sum_{j=1}^{s} n_{j} \mathbf{y}_{j}
$$

with all $n_{j}=n_{j}(\mathbf{v}) \in \mathbb{Z}$. Since $\Lambda$ is an $(r, R)$-set there is a vector $\tilde{\mathbf{w}}_{0} \in \Lambda$ with $\left\|\tilde{\mathbf{w}}_{0}-\mathbf{v}\right\| \leqq R$. We call $\tilde{\mathbf{w}}_{0}$ the reference vector for $\mathbf{v}$. We will prove that

$$
\mathbf{v}=\tilde{\mathbf{w}}_{0}+\mathbf{f}, \quad \text { some } \mathbf{f} \in F .
$$

Since $\mathbf{0} \in \Lambda$, we have $\Lambda \subseteq \Lambda-\Lambda \subseteq \mathbb{Z}\left[\mathbf{y}_{1}, \ldots, \mathbf{y}_{s}\right]$, hence

$$
\tilde{\mathbf{w}}_{0}=\sum_{j=1}^{s} \tilde{n}_{j}^{(0)} \mathbf{y}_{j}
$$

with all $\tilde{n}_{j}^{(0)} \in \mathbb{Z}$. Thus (3.6) is equivalent to

$$
\left|n_{j}-{\tilde{n_{j}}}^{(0)}\right| \leqq 2 C_{1}\left(\frac{4 R}{r^{\prime}}+1\right)^{n}, \quad \text { for } 1 \leqq j \leqq s .
$$

We deform the reference vector $\tilde{\mathbf{w}}_{0}=\tilde{\mathbf{w}}_{0}-\mathbf{0}$ to the vector $\mathbf{v}=\tilde{\mathbf{w}}-\mathbf{w}$ by a series of steps drawn from $\mathscr{S}-\mathscr{S}$. Introduce the vectors

$$
\begin{cases}\mathbf{z}_{k}=k R \frac{\mathbf{w}}{\|\mathbf{w}\|}, & \text { for } 0 \leqq k \leqq k_{0}=\left\lfloor\frac{\|\mathbf{w}\|}{R}\right\rfloor, \\ \tilde{\mathbf{z}}_{k}:=\mathbf{z}_{k}+\mathbf{v}, & \text { for } 0 \leqq k \leqq k_{0},\end{cases}
$$


By the Delaunay property of $\Lambda$ there exist vectors $\mathbf{w}_{k}, \tilde{\mathbf{w}}_{k} \in \Lambda$ with

$$
\left|\tilde{\mathbf{w}}_{k}-\tilde{\mathbf{z}}_{k}\right| \leqq R, \quad\left|\mathbf{w}_{k}-\mathbf{z}_{k}\right| \leqq R, \quad \text { for } 1 \leqq k \leqq k_{0}
$$

The vectors $\mathbf{w}_{0}=\mathbf{0}$ and the reference vector $\tilde{\mathbf{w}}_{0}$ satisfy this property for $k=0$, and so do the vectors

$$
\tilde{\mathbf{w}}_{k_{0}+1}:=\tilde{\mathbf{w}}, \quad \mathbf{w}_{k_{0}+1}:=\mathbf{w}
$$

for $k=k_{0}+1$. Now define

$$
\mathbf{v}_{k}:=\tilde{\mathbf{w}}_{k}-\mathbf{w}_{k}, \quad \text { for } 0 \leqq k \leqq k_{0}+1
$$

so that $\mathbf{v}_{0}=\tilde{\mathbf{w}}_{0}$ and $\mathbf{v}_{k_{0}+1}=\mathbf{v}$. Since $\mathbf{v}_{k} \in \Lambda-\Lambda$, we have

$$
\mathbf{v}_{k}=\sum_{j=1}^{s} n_{j}^{(k)} \mathbf{y}_{j}
$$

for some $n_{j}^{(k)} \in \mathbb{Z}$. We next show that $\mathbf{v}_{k}-\mathbf{v}_{k-1} \in \mathscr{S}-\mathscr{S}$. Now

$$
\mathbf{v}_{k}-\mathbf{v}_{k-1}=\left(\tilde{\mathbf{w}}_{k}-\tilde{\mathbf{w}}_{k-1}\right)-\left(\mathbf{w}_{k}-\mathbf{w}_{k-1}\right),
$$

and a triangle inequality argument as in (3.4) gives

$$
\begin{aligned}
& \left\|\tilde{\mathbf{w}}_{k}-\tilde{\mathbf{w}}_{k-1}\right\| \leqq 3 R, \quad 1 \leqq k \leqq k_{0}+1, \\
& \left\|\mathbf{w}_{k}-\mathbf{w}_{k-1}\right\| \leqq 3 R, \quad 1 \leqq k \leqq k_{0}+1 \text {, }
\end{aligned}
$$

hence $\tilde{\mathbf{w}}_{k}-\tilde{\mathbf{w}}_{k-1} \in \mathscr{S}$ and $\mathbf{w}_{k}-\mathbf{w}_{k-1} \in \mathscr{S}$. Thus $\mathbf{v}_{k}-\mathbf{v}_{k-1} \in \mathscr{S}-\mathscr{S}$, and the bound (3.2) applied to $\mathbf{v}_{k}-\mathbf{v}_{k-1}$ gives

$$
\left|n_{j}^{(k)}-n_{j}^{(k-1)}\right| \leqq 2 C_{1}, \quad 1 \leqq j \leqq s .
$$

Now we show that all $\mathbf{v}_{j}$ are close to $\mathbf{v}$. Using $\mathbf{v}=\tilde{\mathbf{z}}_{j}-\mathbf{z}_{j}$ and the triangle inequality gives

$$
\left\|\mathbf{v}_{j}-\mathbf{v}\right\|=\left\|\left(\tilde{\mathbf{w}}_{j}-\mathbf{w}_{j}\right)-\left(\tilde{\mathbf{z}}_{j}-\mathbf{z}_{j}\right)\right\| \leqq\left\|\tilde{\mathbf{w}}_{j}-\tilde{\mathbf{z}}_{j}\right\|+\left\|\mathbf{w}_{j}-\mathbf{z}_{j}\right\| \leqq 2 R .
$$

Thus all the points $\mathbf{v}_{j}$ lie in the ball $B(\mathbf{v}, 2 R)$ of radius $2 R$ around $\mathbf{v}$, and this ball contains at most $\left(\frac{4 R}{r^{\prime}}+1\right)^{n}$ elements of $\Lambda-\Lambda$ by the same argument as (3.1).

To complete the proof, we suppose that the bound (3.7) does not hold and argue by contradiction. The sequence of vectors $\mathbf{v}_{k}$ starts from the reference vector $\mathbf{v}_{0}=\tilde{\mathbf{w}}_{0}$ and ends with $\mathbf{v}_{k_{0}+1}=\mathbf{v}$, and takes steps that are bounded in the sense (3.10). If (3.7) does not hold, then there must be among $1 \leqq j \leqq s$ some coordinate position $j_{0}$ such that

$$
\left|n_{j_{0}}^{\left(k_{0}+1\right)}-n_{j_{0}}^{(0)}\right|>2 C_{1}\left(\frac{4 R}{r^{\prime}}+1\right)^{n}
$$

The bound (3.10) implies that over $0 \leqq k \leqq k_{0}+1$ the coefficient $n_{j_{0}}^{(k)}$ must take on at least $\left(\frac{4 R}{r^{\prime}}+1\right)^{n}+1$ distinct values, so that at least this many points $\mathbf{v}_{k}$ are distinct. This contradicts the upper bound $\left(\frac{4 R}{r^{\prime}}+1\right)^{n}$ for the number of points in $\Lambda-\Lambda$ in the ball $B(\mathbf{v}, 2 R)$, proved similarly to (3.1). 


\section{Harmonious Set Criterion to be a Meyer Set}

We deduce from Theorem 1.1 an improvement of the harmonic-analytic criterion (3) of Theorem 2.1 for a set $\Lambda$ to be a Meyer set. This result and its proof are due to Robert Moody.

Theorem 4.1. The following properties of a set $\Lambda$ in $\mathbb{R}^{n}$ are equivalent.

(1) $A$ is a Meyer set.

(2) $\Lambda$ is relatively dense and there is some $\varepsilon$ with $0<\varepsilon<\frac{1}{2}$ such that $\Lambda^{\varepsilon}$ is relatively dense, i.e. such that $\Lambda$ is $\varepsilon$-harmonious.

To prove this result we begin with the following lemma.

Lemma 4.1. If a set $\Lambda$ in $\mathbb{R}^{n}$ is relatively dense, then for $0<\varepsilon<1$ its $\varepsilon$-dual $\Lambda^{\varepsilon}$ is uniformly discrete.

Proof. The relative denseness of $\Lambda$ means that $\Lambda+B(\mathbf{0}, R)=\mathbb{R}^{n}$ for some finite $R$. We argue by contradiction. Suppose that $0<\varepsilon<1$ and that $\Lambda^{\varepsilon}$ is not uniformly discrete. We can then find two sequences of points $\boldsymbol{\mu}_{i}^{(1)}, \boldsymbol{\mu}_{i}^{(2)} \in \Lambda^{\varepsilon}$, with $\boldsymbol{\mu}_{i}^{(1)} \neq \boldsymbol{\mu}_{i}^{(2)}$ and $\left\|\boldsymbol{\mu}_{i}^{(1)}-\boldsymbol{\mu}_{i}^{(2)}\right\| \rightarrow 0$ as $i \rightarrow \infty$, such that

$$
\| \exp \left\langle 2 \pi i\left\langle\boldsymbol{\mu}_{i}^{(j)}, \mathbf{x}\right\rangle-1 \|<\varepsilon, \quad \text { for all } \mathbf{x} \in \Lambda, j=1,2 .\right.
$$

This yields

$$
\begin{aligned}
& \left\|\exp \left(2 \pi i\left\langle\boldsymbol{\mu}_{i}^{(1)}-\boldsymbol{\mu}_{i}^{(2)}, \mathbf{x}\right\rangle\right)-1\right\|=\left\|\exp \left(2 \pi i\left\langle\boldsymbol{\mu}_{i}^{(1)}, \mathbf{x}\right\rangle\right)-\exp \left(2 \pi i\left\langle\boldsymbol{\mu}_{i}^{(2)}, \mathbf{x}\right\rangle\right)\right\| \\
& \quad \leqq \sum_{j=1}^{2}\left\|\exp \left(2 \pi i\left\langle\boldsymbol{\mu}_{i}^{(2)}, \mathbf{x}\right\rangle\right)-1\right\|<2 \varepsilon, \quad \text { for all } \mathbf{x} \in \Lambda
\end{aligned}
$$

Since $\varepsilon<1$, we have $\left\|\boldsymbol{\mu}_{i}^{(1)}-\boldsymbol{\mu}_{i}^{(2)}\right\|<\frac{1-\varepsilon}{8 R}$ for all sufficiently large $i$, which yields

$$
\begin{aligned}
& \left\|\exp \left(2 \pi i\left\langle\boldsymbol{\mu}_{i}^{(1)}-\boldsymbol{\mu}_{i}^{(2)}, \mathbf{x}\right\rangle\right)-1\right\| \\
& \quad \leqq\left\|\exp \left(\frac{\pi i}{4}(1-\varepsilon)\right)-1\right\|<2-2 \varepsilon, \quad \text { all } \mathbf{x} \in B(\mathbf{0}, R) .
\end{aligned}
$$

It follows from (4.2) and (4.3) that

$$
\left\|\exp \left(2 \pi i\left\langle\boldsymbol{\mu}_{i}^{(1)}-\boldsymbol{\mu}_{i}^{(2)}, \mathbf{x}\right\rangle\right)-1\right\|<2, \quad \text { all } \mathbf{x} \in \mathbb{R}^{n},
$$

which implies that $\boldsymbol{\mu}_{i}^{(1)}=\boldsymbol{\mu}_{i}^{(2)}$, a contradiction that proves the lemma.

Lemma 4.1 is sharp in the sense that for any given $\varepsilon>1$ there exists a Delone set $\Lambda$ such that its $\varepsilon$-dual $\Lambda^{\varepsilon}$ is not uniformly discrete.

Proof of Theorem 4.1 The implication (1) $\Rightarrow$ (2) follows from Theorem 2.1.

To prove (2) $\Rightarrow(1)$, suppose that $\Lambda$ is relatively dense and that $\Lambda^{\varepsilon}$ is relatively dense for some fixed $\varepsilon$ with $0<\varepsilon<\frac{1}{2}$. Now (4.2) shows that $\Lambda^{\varepsilon}-\Lambda^{\varepsilon} \subseteq \Lambda^{2 \varepsilon}$. Next, since $2 \varepsilon<1$, Lemma 4.1 shows that $\Lambda^{2 \varepsilon}$ is uniformly discrete. Since a translate $\Lambda^{\varepsilon}-\{\boldsymbol{\mu}\} \subseteq \Lambda^{\varepsilon}-\Lambda^{\varepsilon} \subseteq \Lambda^{2 \varepsilon}$, it follows that $\Lambda^{\varepsilon}$ is uniformly discrete, hence $\Lambda^{\varepsilon}$ is a Delone set. In addition $\Lambda^{\varepsilon}-\Lambda^{\varepsilon}$ is relatively dense because it contains $\Lambda^{\varepsilon}-\{\boldsymbol{\mu}\}$ for some $\mu$, hence $\Lambda^{\varepsilon}-\Lambda^{\varepsilon}$ is also a Delone set. Theorem 1.1 now applies to show that $\Lambda^{\varepsilon}$ is a Meyer set. 
Next, we study $\Lambda^{\varepsilon \varepsilon}=\left(\Lambda^{\varepsilon}\right)^{\varepsilon}$. Certainly $\Lambda \subseteq \Lambda^{\varepsilon \varepsilon}$, hence $\Lambda^{\varepsilon \varepsilon}$ is relatively dense. Thus $\Lambda^{\varepsilon}$ and $\left(\Lambda^{\varepsilon}\right)^{\varepsilon}$ are both relatively dense, so the argument above applies to $\Lambda^{\varepsilon}$ to show that $\Lambda^{\varepsilon \varepsilon}$ is a Meyer set. Now $\Lambda$ inherits uniform discreteness from $\Lambda^{\varepsilon \varepsilon}$ because $\Lambda \subseteq \Lambda^{\varepsilon \varepsilon}$, hence $\Lambda$ is a Delone set. In addition $\Lambda-\Lambda \subseteq \Lambda^{\varepsilon}-\Lambda^{\varepsilon} \subseteq\left(\Lambda^{\varepsilon}\right)^{2 \varepsilon}$ is uniformly discrete by Lemma 4.1 , hence $\Lambda-\Lambda$ is a Delone set. Now Theorem 1.1 implies that $\Lambda$ is a Meyer set.

This proof also establishes the following result.

Corollary 4.1. If $\Lambda$ is a Meyer set, then $\Lambda^{\varepsilon}$ is a Meyer set for $0<\varepsilon<\frac{1}{2}$.

Robert Moody has shown that the conclusion of Corollary 4.1 is actually true for the range $0<\varepsilon<2$, by another method.

\section{Tilings and Meyer Sets}

Various tiling models for quasicrystals can be incorporated into the framework of quasiregular sets. We consider tilings using copies of a finite set $\mathscr{P}$ of prototiles, each of which is a simply connected bounded polytope. ${ }^{2}$ A tiling of $\mathbb{R}^{n}$ by $\mathscr{P}$ is a covering of $\mathbb{R}^{n}$ with copies of the prototiles moved by isometries, in which the interiors of all copies are disjoint. A translation tiling by $\mathscr{P}$ is the same except that only translations of prototiles are allowed. We can associate a Delone set $\Lambda$ to a tiling by putting a fixed mark in the interior of each prototile, with $\Lambda$ representing the locations of the marks in the tiling using these prototiles. Different prototiles are assigned different colored marks to distinguish them. Let $\Lambda_{i}$ be the locations of the marks of color $i$ in a tiling and let $\Lambda=\bigcup \Lambda_{i}$ be the locations of all marks.

Tilings are typically prescribed by a finite set of local rules for allowable neighborhoods $(\Lambda-\mathbf{x}) \cap B(\mathbf{0}, R)$ for $\mathbf{x} \in \Lambda_{i}$, for each color class $i$. Such local rules may prescribe decorations or matching rules added to the tiles. A legal tiling is one that satisfies the local rules. Now consider local rules under translations. Legal tilings for local rules under translations always have the locally finite atlas property. For any tiling having the locally finite atlas property, it can be shown that any associated set $\Lambda$ of marked points has $\Lambda \subseteq \mathbb{Z}\left[\mathbf{v}_{1}, \mathbf{v}_{2}, \ldots, \mathbf{v}_{s}\right]$ for some finite set of vectors $\left\{\mathbf{v}_{i}: 1 \leqq i \leqq s\right\}$. We do not know what extra assumptions, if any, are needed on such tilings to make $\Lambda$ a quasiregular set. However "cut-and-project" constructions of quasiperiodic tilings (as in [29]) have the property that any set of marks $\Lambda$ as well as each $\Lambda_{i}$ separately will be quasiregular sets. (We note that tilings given by local rules under isometries need not have the locally finite atlas property, e.g. the pinwheel tilings of Radin [36].)

In the converse direction, a Delone set $\Lambda$ gives rise to a partition $\mathscr{T}$ of $\mathbb{R}^{n}$ into polytopes $\{V(\mathbf{x}): \mathbf{x} \in \Lambda\}$ in which $V(\mathbf{x})$ is the Voronoi cell (or Dirichlet domain) of $\mathbf{x} \in \Lambda$, namely

$$
V(\mathbf{x}):=\left\{\mathbf{y} \in \mathbb{R}^{n}:\|\mathbf{y}-\mathbf{x}\| \leqq\left\|\mathbf{y}-\mathbf{x}^{\prime}\right\| \text { for all } \mathbf{x}^{\prime} \in \Lambda\right\} .
$$

If $\Lambda$ has the locally finite atlas property, then the Voronoi cells $V(\mathbf{x})$ will have a finite number of distinct shapes (up to isometries), and thus $\mathscr{T}$ is a translation tiling of $\mathbb{R}^{n}$ using a finite set of prototiles. In particular, a quasiregular set $\Lambda$ gives rise to a tiling with a finite set of prototiles.

\footnotetext{
${ }^{2}$ More generally, each prototile is a simply connected compact set which is the closure of its interior and has a boundary of measure zero.
} 
Meyer sets include many reasonable quasicrystal structures. However there are Meyer sets that do not correspond to any quasicrystal structure in the sense that they do not have a well-defined autocorrelation function or limiting X-ray diffraction pattern, as defined in Hof [17]. Take a lattice $L$ and a finite set $F$ containing at least two elements and with $L \cap F=\emptyset$. Then any $\Lambda$ with $L \subseteq \Lambda \subseteq L+F$ is a Meyer set. The local density of $L$ is $|\operatorname{det} L|^{-1}$, and that of $L+F$ is $|F||\operatorname{det} L|^{-1}$. Clearly we can choose $\Lambda$ to have a density in $\Lambda \cap B(\mathbf{0}, R)$ that oscillates between these two limits as $R \rightarrow \infty$. Thus $\Lambda$ has no limiting density, and we can further arrange that $\Lambda$ has no limiting autocorrelation function in the sense of Hof $[16,18]$. It follows that Meyer sets are more general than a concept of "quasicrystal" which requires a nice X-ray diffraction pattern.

One may seek a narrower concept of "ideal quasicrystal" that consists of a subclass of Meyer sets satisfying additional conditions. Three possibilities for such additional conditions include restricting to Meyer sets that are self-dual in the sense $\Lambda^{\varepsilon \varepsilon}=\Lambda$ as discussed in [33], or restricting to Meyer sets that have an autocorrelation function which is a countable sum of weighted delta-functions, or restricting to Meyer sets which satisfy a set of local rules under translation. This last notion appears to contain the "tiling dynamical systems" of Robinson [38] and Solomyak [43], and is also closely related to viewpoints of Radin [35,37] using "shifts of finite type."

Acknowledgements I am indebted to M Bakke, R. Moody and M. Senechal for helpful comments and references, and also to R. Moody for permission to include his results in Sect. 4.

\section{References}

1 Bombieri, E., Taylor, J.E.: Which distributions diffract? An initial investigation. J. Phys. Colloq 47, C3, 19-28 (1986)

2. de Bruijn, N G.: Algebraic theory of Penrose's nonperiodic tiling of the plane I, II. Nederl Akad Wetensch. Proc Series A 84, 39-52 and 53-66 (1981)

3 de Bruijn, N.G: Quasicrystals and their Fourier transform. Nederl. Akad. Wetensch Proc Ser A 89, 123-152 (1986)

4. Burkov, S.E : Absence of weak local rules for the planar quasicrystallographic tiling with 8-fold symmetry. Commun. Math. Phys. 117, 667-675 (1988)

5 Chen, L., Moody, R.V , Patera, J : Non-crystallographic root systems and quasicrystals. Preprint

6 Delaunay, B.N., Delone, B.N : Neue Darstellung der Geometrischen Kristallographie Zeit. Kristallographie 84, 109-149 (1932)

7. Delone, B.N., Dolbilin, N.P., Štogrin, M I, Galiulin, R V : A local criterion for regularity in a system of points Sov. Math. Dokl. 17, 319-322 (1976)

8 Dolbilin, N.P., Lagarias, J.C., Senechal, M : Multiregular Point Systems. Preprint

9 Duneau, M., Katz, A.: Quasiperiodic structures Phys Rev. Lett. 54, 2688-2691 (1985)

10 Elser, V : The diffraction pattern of projected structures. Acta. Cryst. A 42, 36-43 (1986)

11 Engel, P : Geometric Crystallography - An Axiomatic Introduction to Crystallography. Reidel: Dordrecht, 1986

12. Engel, P.: Geometric Crystallography In: Handbook of Convex Geometry, Volume B P. Gruber and J M Wills (eds ) Amsterdam: North-Holland, 1993, pp. 991-1041

13 Gähler, F., Rhyner, J.: Equivalence of the generalized grid and projection methods for the construction of quasiperiodic tilings. J. Phys A 19, 267-277 (1986)

14. Galiulin, R V : Delone systems. Sov. Phys. Crystallogr 25, No 5, 517-521 (1980)

15. Galiulin, R.V.: Zonohedral Delone systems. In: Collected Abstracts. XII European Crystallog. Meeting, Moscow, Vol. I 1989, p. 21

16 Hof, A.: Quasicrystals, Aperiodicity and Lattice Systems. Thesis, U of Groningen, 1992 
17. Hof, A.: On diffraction by aperiodic structures. Commun. Math. Phys., 174, 149-159, 1995

18. Hof, A.: Diffraction by aperiodic structures at high temperatures. J. Phys. A: Math Gen. 28, 57-62 (1995)

19. Janot, C.: Quasicrystals: A Primer. Oxford: Oxford University Press, 1992

20 Katz, A.: Theory of matching rules for 3-dimensional Penrose tilings. Commun. Math. Phys. 119, 262-268 (1988)

21. Katz, A., Duneau, M.: Quasiperiodic structures determined by the projection method. J. Phys. (Paris) Supp. C47, 103-112 (1987)

22. Kramer, P.: Non-periodic central space filings with icosahedral symmetry using copies of seven elementary cells. Acta Cryst. A 38, 257-264 (1982)

23. Kramer, P., Neri, R.: On periodic and nonperiodic space fillings of $\mathbb{E}^{n}$ obtained by projection. Acta Cryst. A 40, 580-587 (1984) (Erratum: Acta Cryst. A 41, 619 (1985))

24. Kramer, P., Papadopolis, Z., Moody, R.V.: A growth mechanism for the $T^{(2 F)}$ tiling. Preprint

25. Le, T.Q.T.: Local rules for pentagonal quasicrystals. Disc. \& Comp. Geom. 14, 31-70 (1995)

26. Le, T.Q.T., Plunikhin, S., Sadov, V.: Geometry of quasicrystals. Uspeki Math. Nauk. 48, 41-102 (1993) (in Russian). English translation: Russian Math. Surveys 48, 37-100 (1993)

27. Levine, D., Steinhardt, P.J.: Quasicrystals: A new class of ordered structures. Phys Rev Lett 53, 2477-2480 (1984)

28. Levitov, L.S.: Local rules for quasicrystals. Commun. Math. Phys. 119, 627-666 (1988)

29. Lunnon, W.F., Pleasants, P.A.B.: Quasicrystallographic tilings. J. Maths. Pures Appl. 66, 217-263 (1987)

30 Meyer, Y.: Nombres de Pisot, Nombres de Salem, et analyse harmonique. Lecture Notes in Math. No. 117, Berlin, Heidelberg, New York: Springer, 1970

31. Meyer, Y.: Algebraic Numbers and Harmonic Analysis. Amsterdam: North-Holland, 1972

32. Meyer, Y.: Quasicrystals, Diophantine Approximation and Algebraic Numbers. In: Beyond Quasicrystals. F. Axel, D. Gratias (eds.) Les Editions de Physique. Berlin, Heidelberg, New York: Springer, 1995, pp. 3-16

33. Moody, R.V.: Meyer Sets and the Finite Generation of Quasicrystals. In: Symmetries in Science VIII. B. Gruber (ed.) London: Plenum, 1995

34 Moody, R. V., Patera, J.: Local dynamical generation of quasicrystals. Preprint

35. Radin, C.: Global order from local sources. Bull. Am. Math. Soc. 25, 335-364 (1991)

36. Radin, C.: The pinwheel tilings of the plane. Ann. Math. 139, 661-702 (1994)

37. Radin, C.: Space tilings and substitutions. Geometriae Dedicata 55, 257-264 (1995)

38. Robinson, E.A., Jr.: The Dynamical Theory of Tilings and Quasicrystallography. In: Multidimensional Symbolic Dynamics: Proceedings of the Special Year Warwick 1994

39. Senechal, M.: Quasicrystals and Geometry. Cambridge: Cambridge University Press, 1995

40. Shechtman, D., Blech, I., Gratias, D, Cahn, J.W.: Metallic phase with long-range orientational order and no translational symmetry. Phys. Rev. Lett. 53, 1951-1953 (1984)

41. Socolar, J.: Weak matching rules for quasicrystals. Commun. Math Phys. 129, 599-619 (1990)

42. Sohncke, L.: Die regelmässigen ebenen Punktsysteme von unbegrenzter Ausdehnung. J. reine Angew. 77, 47-101 (1874)

43. Solomyak, B.: Tiling dynamical systems. Ergod. Th.\&Dyn. Sys., to appear

Communicated by J.L Lebowitz 